\title{
La integración de información contextual en el discurso de personas con demencia
}

\author{
ANa Varela SuÁRez \\ Facultade de Filoloxía e Tradución \\ Universidade de Vigo (Vigo) \\ Departamento de Tradución e Lingüística \\ Campus Lagoas Marcosende \\ 36310 Vigo \\ E-mail: anavarela@uvigo.es
}

\section{LA INTEGRACIÓN DE INFORMA- CIÓN CONTEXTUAL EN EL DIS- CURSO DE PERSONAS CON DE- MENCIA}

RESUMEN: Este trabajo tiene como objetivos: (i) proponer un modelo de análisis de la integración de información contextual en el discurso; (ii) identificar cuáles son los patrones de error de las personas con demencia cuando integran la información contextual en su discurso; (iii) comprobar si la capacidad de las personas con demencia para introducir la información contextual correctamente se reduce conforme avanza el deterioro cognitivo. Para ello se ha propuesto un modelo teórico y se ha aplicado al análisis de un corpus, formado por grabaciones en vídeo de 23 informantes con distintos tipos de demencia mientras conversaban sobre sus rutinas, gustos e historia personal. El modelo propuesto ha funcionado correctamente como herramienta de análisis de nuestro corpus. Los resultados indican que existe una correlación negativa fuerte entre el estadio de la enfermedad y la capacidad para integrar la información contextual en los enunciados de las personas con demencia.

PALABRAS CLAVES: contexto pragmática; coherencia; demencia; Alzheimer.

SUMARIO: 1. Introducción. 2. Estado de la cuestión. 3. Marco teórico. 4. Materiales y métodos. 5. Resultados. 6. Discusión y conclusiones.
THE INTEGRATION OF CONTEXTUAL INFORMATION IN THE DISCOURSE OF PEOPLE WITH DEMENTIA

ABSTRACT: This study pursues the following objectives: (i) developing a model for analyzing the integration of contextual information in discourse; (ii) identifying the patterns of error committed by people with dementia when they integrate contextual information in their discourse; (iii) verifying whether the ability of people with dementia to correctly use contextual information decreases as the cognitive impairment advances. In order to do so, a theoretical model has been proposed and it has been applied for analyzing a corpus, consisting of video recordings of 23 people with dementia while talking about their routines, likes, dislikes and personal history. The model has proved to work correctly as an analysis tool for our corpus. The results show that there exists a strong correlation between the ability to integrate contextual information of our informants and the stage of the disease in which they are.

KEY WORDS: context; pragmatics; coherence; dementia; Alzheimer.

SUMMARY: 1 . Introduction. 2 . State of the art. 3. Theoretical framework. 4. Methods and materials. 5. Results. 6. Discussion and conclusions.

\section{L'INTEGRATION D'INFORMATION CONTEXTUELLE DANS LE DISCOURS DE PERSONNES AVEC DÉMENCE}

RÉSUMÉ: Ce travail présente les objectifs suivants: (i) développer un modèle pour analyser l'intégration d'information contextuelle dans le discours; (ii) identifier les patrons d'erreur que commentent les personnes souffrant de démence quand elles intègrent l'information contextuelle dans le discours; (iii) vérifier si la capacité d'utiliser correctement l'information contextuelle se réduit à mesure qu'avance le déclin cognitif. Pour ce faire, on a proposé un modèle théorique et on l'a appliqué pour analyser un corpus, composé de vidéos de 23 personnes souffrant de démence qui parlent de leurs routines, goûts et histoire personnelle. Le modèle a fonctionné correctement comme outil d'analyse de notre corpus. Les résultats montrent qu'il existe une corrélation forte entre la capacité d'intégrer l'information contextuelle des participants et le stade de leur démence.

MOTS CLÉS: $\begin{array}{r}\text { contexte, } \\ \text { pragmatique; } \\ \text { démence; Alzheimer. }\end{array}$

SOMMAIRE: 1 . Introduction. 2 État de l'art. 3. Cadre théorique. 4. Méthodologie. 5. Résultats. 6. Discussion et conclusions.

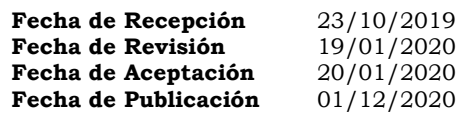

Fecha de Recepción Fecha de Aceptación $01 / 12 / 2020$ 


\section{La integración de información contextual en el discurso de personas con demencia}

\section{ANA VARELA SUÁREZ}

\section{INTRODUCCIÓN}

Actualmente, hay 47,5 millones de personas diagnosticadas con demencia en el mundo, lo que representa entre un $5 \%$ y un $8 \%$ del total de las personas mayores de 60. Esta enfermedad produce deterioro cognitivo en las áreas de la memoria, orientación, cálculo, pensamiento, juicio, capacidad de aprendizaje y lenguaje, tanto a nivel de comprensión como de producción, de forma más acuciada de lo que se considera estándar en un envejecimiento normal (Organización Mundial de la Salud, 2017).

Muchos de los trabajos realizados hasta ahora en el ámbito de lenguaje y demencia señalan que una de las áreas en las que se detecta un deterioro significativo es en la de pragmática (véase, entre otros, a Hamilton, 1994; Ramanathan, 1995; Nebes y Halligan, 1996; Joubert et al. 2004; Asp, y de Villiers, 2010; Pérez Mantero, 2017; Varela Suárez, 2018). Puesto que la pragmática estudia el uso de la lengua en contexto, en este trabajo hemos querido centrarnos en este aspecto y estudiar cómo las personas que padecen demencia introducen la información contextual en su discurso. Con esto en mente, esta investigación presenta los siguientes objetivos:

(i) Proponer un modelo de análisis de la integración de información contextual en el discurso.

(ii) Identificar cuáles son los patrones de error de las personas con demencia cuando integran la información contextual en su discurso.

(iii) Comprobar si la capacidad de las personas con demencia para introducir la información contextual correctamente se reduce conforme avanza el deterioro cognitivo.

\section{ESTADO DE LA CUESTIÓN}

El número trabajos dedicados a las habilidades pragmáticas de las personas que tienen demencia es elevado, por lo que en este apartado hemos optado por limitarnos exclusivamente a aquellas aportaciones centradas en cómo influye la información contextual a la producción y comprensión del discurso de esta población. Puesto que la Enfermedad de Alzheimer (EA) es la forma de demencia más común, ya que supone entre el $60-70 \%$ de los casos (OMS, 2017), las investigaciones mencionadas en este apartado se centran en esta enfermedad.

En primer lugar, es necesario comentar que, dado que en el campo de la pragmática clínica no existe un consenso sobre cuál es la definición de contexto sobre la que debemos partir, la mayor parte de los trabajos realizados 
dentro de esta área de estudio han partido de perspectivas ligeramente diferentes (Cummings, 2012b). La primera aportación que queremos mencionar es Ramanathan-Abbott (1994), que concluyó que, aunque las personas con EA padecen trastornos discursivos severos, mantienen la capacidad de seguir conversaciones extensas y con sentido en función de la interacción social. En su siguiente estudio, Ramanathan (1995) comprobó que el contexto en el que tiene lugar la interacción social es clave para que la comunicación sea exitosa y resaltó la importancia de que el análisis lingüístico de las personas que padecen esta enfermedad siempre se realice desde un enfoque en el que se tengan en cuenta las circunstancias en las que se produzca la interacción.

El trabajo de Nebes y Halligan (1996) está enfocado desde una perspectiva más semántica y concluyó que la activación de los atributos en el discurso de las personas con EA se ve afectada por los elementos contextuales y que se deteriora conforme avanza la enfermedad. También dentro del ámbito semántico, Chenery et al. (1990) plantearon a un grupo de control y otro con EA una serie de palabras homófonas y les pidieron que determinasen si estas encajaban o no en un cierto contexto discursivo midiendo dos intervalos de estímulo: 330ms y $1000 \mathrm{~ms}$. El resultado fue que las personas con EA mostraron alteraciones en la activación selectiva automática del material léxico así como en la integración y creación del material léxico dentro del discurso que estaban escuchando.

El último trabajo que nos gustaria resaltar es el de Joubert et al. (2004), que examinó a una mujer con EA a la que pidieron que identificase a sus familiares y a personas famosas en fotografias. En una primera ronda, ayudaron a la informante planteándole un contexto espacial y temporal, pero en la segunda retiraron esta ayuda. Los resultados de este trabajo indican que la mujer era capaz de identificar a sus familiares con y sin ayuda de los elementos contextuales, pero no pudo recordar el nombre de ninguna de las personas.

\section{MARCO TEÓRICO}

De acuerdo con Van Dijk y Kintsch (1983), además de una representación del significado de un texto, los usuarios de una lengua también construyen modelos mentales de los eventos que se incluyen en un texto; es decir, reconstruyen mentalmente las situaciones a las que se refieren. Van Dijk (2012) propone que estos modelos mentales son únicos, personales y subjetivos, pero que están delimitados por un lado por el escenario (tiempo y lugar) en el que la interacción tiene lugar; y por otro, por los participantes (roles y situaciones) y acciones. Para poder realizar estudios sobre el contexto, como en el caso de este trabajo, Van Dijk propone una subcategoría de modelo mental: "contextual models which organize the ways in which our speech is strategically structured and adapted to every communicative situation" (2012: 116). 
Partiendo de esto, Van Dijk (2012: 76) también presenta un esquema de modelo contextual básico:

- $\quad$ "Setting: Time/Period, Space/Place/Environment;

- Participants (self, others);

- communicative roles (participation structure);

- social roles types, membership or identities;

- relations between participants (e.g. power, friendship);

- shared and social knowledge and beliefs;

o intentions and goals;

- Communicative and other Actions/Events".

Este modelo es muy completo y se ajusta a los requisitos de este trabajo para llevar a cabo un análisis de la información contextual en el discurso, por lo que para nuestro análisis hemos adaptado este esquema a nuestras necesidades de anotación y a la realidad de las personas con demencia y lo hemos reconfigurado de la siguiente forma:

- Escenario:

- Tiempo/periodo.

- Espacio/lugar/ambiente.

- Participantes:

- Roles sociales / relaciones entre participantes

- Conocimiento compartido

○ Perfil neuropsicológico.

A partir de esta propuesta, hemos diseñado un sistema de anotación para nuestro corpus que nos ha permitido analizar cómo las personas con demencia introducen la información contextual en el discurso. Este sistema se describe detalladamente en $\$ 4.3$.

\section{MATERIALES Y MÉTODOS}

Para poder cumplir los objetivos de este estudio y analizar la integración de elementos contextuales en el discurso, se decidió recopilar, anotar y analizar un corpus constituido por entrevistas personales a personas que padecían distintos tipos de demencia. En los apartados que siguen, se describe cómo se han realizado cada uno de estos pasos.

\subsection{Participantes}

El conjunto de participantes de este trabajo se reunió gracias a la colaboración de la Asociación de Familiares y Enfermos de Alzheimer de Galicia (AFAGA), que nos ofreció sus instalaciones para poder realizar las entrevistas y nos entregó un informe con una valoración neuropsicológica de cada 
una de las personas. En total, la muestra de este estudio constó de 23 personas, 17 de ellas con Enfermedad de Alzheimer, 3 con demencia mixta, 1 con demencia vascular, 1 con demencia frontotemporal y 1 sin especificar. De ellas, 12 se encontraban en el estadio inicial de la enfermedad (GDS-3, GDS-4), 6 en el estadio moderado (GDS-5) y 5 en el estadio severo (GDS-6 y GDS-7). Además, el $60 \%$ tenían como primera lengua el castellano, frente el $40 \%$ que tenían el gallego, y el $60 \%$ eran mujeres, frente al $40 \%$ que eran hombres. La edad media de la muestra fue de 80 años (Desviación Estándar 8). La Tabla 1 indica los datos de todas las personas participantes:

\begin{tabular}{|c|c|c|c|c|c|}
\hline Informante & Diagnóstico & GDS & Edad & Lengua & Género \\
\hline I01 & E. de Alzheimer & 7 & 90 & Castellano & Mujer \\
\hline I02 & E. de Alzheimer & 7 & 77 & Gallego & Mujer \\
\hline I03 & E. de Alzheimer & 7 & -1 & Castellano & Mujer \\
\hline I04 & Dem. Mixta & 6 & 90 & Gallego & Hombre \\
\hline I05 & E. de Alzheimer & 6 & 61 & Castellano & Hombre \\
\hline I06 & E. de Alzheimer & 5 & 90 & Gallego & Mujer. \\
\hline I07 & E. de Alzheimer & 5 & 86 & Gallego & Mujer \\
\hline $\mathrm{I08}$ & E. de Alzheimer & 5 & 73 & Gallego & Hombre \\
\hline I09 & Dem. Mixta & 5 & 86 & Gallego & Hombre \\
\hline $\mathrm{I} 10$ & Dem. Vascular & 5 & 86 & Castellano & Mujer \\
\hline I1 1 & Dem. sin especificar & 5 & - & Gallego & Mujer \\
\hline $\mathrm{I} 12$ & E. de Alzheimer & 4 & 89 & Castellano & Mujer \\
\hline I13 & E. de Alzheimer & 4 & 83 & Castellano & Mujer \\
\hline I14 & E. de Alzheimer & 4 & 85 & Gallego & Mujer \\
\hline I15 & E. de Alzheimer & 4 & - & Castellano & Mujer \\
\hline I16 & E. de Alzheimer & 4 & 69 & Castellano & Mujer \\
\hline I17 & E. de Alzheimer & 4 & 76 & Castellano & Hombre \\
\hline $\mathrm{I} 18$ & E. de Alzheimer & 4 & - & Castellano & Mujer \\
\hline I19 & E. de Alzheimer & 4 & 80 & Castellano & Hombre \\
\hline $\mathrm{I} 20$ & E. de Alzheimer & 3 & 74 & Castellano & Hombre \\
\hline I2 1 & E. de Alzheimer & 3 & 79 & Castellano & Hombre \\
\hline $\mathrm{I} 22$ & Dem. Frontotemporal & 3 & 79 & Castellano & Mujer \\
\hline I23 & Dem. Mixta & 3 & 72 & Castellano & Mujer \\
\hline
\end{tabular}

Tabla 1: Datos de las personas que han participado en este estudio

\footnotetext{
${ }^{1}$ Los casos en los que no disponemos de la información acerca de la edad de las personas participantes han sido marcados con el símbolo (-) en la tabla.
} 
Antes de ser seleccionadas, todas las personas participantes tuvieron que pasar por los siguientes criterios de inclusión:

a) Presentar un diagnóstico de alguna de las formas de demencia.

b) Aportar una hoja de consentimiento informado, firmado por el cuidador a cargo.

c) Tener como lengua materna el gallego y/o el castellano.

Además, se determinaron los siguientes criterios de exclusión:

a) Padecer déficits audiovisuales que puedan sesgar los resultados de la prueba.

b) Presentar trastornos de la conducta que impidan desarrollar la prueba con normalidad.

c) Presentar un trastorno de afasia severa que dificulte la identificación de palabras y frases para su posterior análisis.

\subsection{RECOGIDA DE DATOS}

Una vez seleccionadas las personas participantes, se les citó en las instalaciones de la AFAGA para realizar la grabación. La prueba consistió en una entrevista personal en la que se les planteó preguntas sobre sus gustos, rutinas e historia personal, con el objetivo de suscitar el lenguaje más natural posible. A continuación, se muestran alguna de las preguntas que se plantearon durante esta tarea:

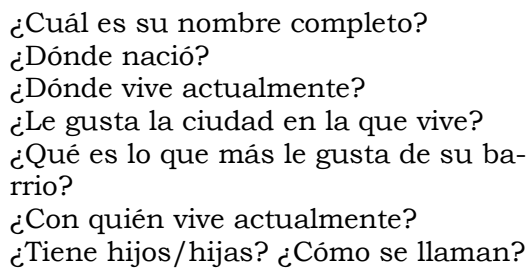

- ¿Tiene nietos/nietas? ¿Cómo se llaman? ¿Cuándo es su cumpleaños? ¿Celebra usted normalmente su cumpleaños?

¿En qué trabajaba usted?

¿Le gustaba su trabajo?

¿Dónde se casó?

Todas las entrevistas fueron grabadas durante abril y mayo de 2016 y las personas participantes hablaron entre 6 y 32 minutos, dependiendo de su predisposición para hablar. Toda la interacción fue recogida en vídeo con una cámara SONY Handycam HD AVCHD, para poder así incluir el lenguaje no verbal en el análisis.

\subsection{TRANSCRIPCIÓN Y ANOTACIÓN}

Tras finalizar las grabaciones, comenzó la fase de transcripción. Todas las grabaciones fueron transcritas utilizando el software libre ELAN. El código empleado fue una adaptación propia de las pautas propuestas por Müller (2006) para la transcripción de textos de personas con trastornos del 
lenguaje. El siguiente paso consistió en un proceso de anotación de fenómenos relacionados con la introducción de información contextual en el discurso. Para ello se siguió el modelo teórico basado en la propuesta de Van Dijk (2012) expuesto en §2 y se establecieron cuatro categorias de análisis: lugar, tiempo, roles sociales y conocimiento compartido. Para cada una de estas categorias se diseñó un sistema de etiquetas de anotación propias, en función de las necesidades de anotación surgidas ad hoc. A continuación se detalla el sistema de etiquetado empleado para cada una de estas categorias:

\section{Lugar}

\begin{tabular}{|c|l|}
\hline Etiqueta & \multicolumn{1}{|c|}{ Explicación } \\
\hline Correcto & $\begin{array}{l}\text { El/la hablante expresa a través de su discurso que es capaz de ubi- } \\
\text { carse espacialmente de forma correcta. } \\
\text { En la calle Zamora; en el Calvario; en Vigo; en Galicia }\end{array}$ \\
\hline Incorrecto & $\begin{array}{l}\text { Expresa en su discurso una ubicación errónea. } \\
\text { Me casé aqui en Palencia. }\end{array}$ \\
\hline Sin referente & $\begin{array}{l}\text { Emplea un deíctico espacial que no tiene referente previo. } \\
\text { Mi infancia fue muy buena. Fui muy feliz allí. }\end{array}$ \\
\hline No específico & $\begin{array}{l}\text { Muestra dudas sobre dónde ha ocurrido un hecho o donde se ubica } \\
\text { una persona u objeto... } \\
\text { Fue en Vigo o en Ourense... Ya no me acuerdo... }\end{array}$ \\
\hline Contradicción & $\begin{array}{l}\text { Presenta una contradicción con respecto al lugar dónde ha ocurrido } \\
\text { un evento o donde se ubica una persona u objeto. } \\
\text { Me casé en Zamora }[. . . \text { Y Yo me casé aqui en Vigo. }\end{array}$ \\
\hline
\end{tabular}

\section{Tiempo}

Tabla 2: Etiquetas de la categoría "lugar"

\begin{tabular}{|c|l|}
\hline Categoria & \multicolumn{1}{|c|}{ Explicación } \\
\hline Correcto & $\begin{array}{l}\text { El/la hablante expresa a través de su discurso que es capaz de ubi- } \\
\text { carse temporalmente de forma correcta. } \\
\text { Hoy; ayer; mañana; el año pasado; el año que viene. }\end{array}$ \\
\hline Incorrecto & $\begin{array}{l}\text { Incluye en su discurso información temporal errónea. } \\
\text { Traballei de camarero e aínda traballo. }\end{array}$ \\
\hline Sin referente & $\begin{array}{l}\text { Emplea un deíctico temporal que no tiene referente previo. } \\
\text { De aquella no me gustaba el pescado, pero ahora sí. }\end{array}$ \\
\hline No específico & $\begin{array}{l}\text { Muestra dudas sobre cuándo ha ocurrido un hecho. } \\
\text { Hace bastante, no recuerdo cuánto. }\end{array}$ \\
\hline Contradicción & $\begin{array}{l}\text { Presenta una contradicción con respecto a cuándo ha ocurrido un } \\
\text { evento. } \\
\text { Me casé cuando me vine a vivir a Vigo. [...] Nos vinimos a Vigo por el } \\
\text { trabajo de mi marido. }\end{array}$ \\
\hline
\end{tabular}

\section{Roles sociales}

Tabla 3: Etiquetas de la categoría "tiempo"

\begin{tabular}{|c|l|}
\hline Categoria & \multicolumn{1}{c|}{ Explicación } \\
\hline Correcto & $\begin{array}{l}\text { El/a hablante reconoce el rol de su interlocutora y se dirige a ella como } \\
\text { tal. } \\
\text { ¿Y esto para qué es? }\end{array}$ \\
\hline Incorrecto & $\begin{array}{l}\text { Confunde a la entrevistadora con otra persona de su entorno. } \\
\text { A ti coñecinche en Lisboa. }\end{array}$ \\
\hline
\end{tabular}




\begin{tabular}{|c|l|}
\hline No relevante & $\begin{array}{l}\text { Emite un enunciado que no es relevante debido a la incapacidad para } \\
\text { reconocer el rol social de la interlocutora. } \\
\text { (Dirigiéndose a la entrevistadora) ¿Puedo darte un besito? }\end{array}$ \\
\hline
\end{tabular}

Tabla 4: Etiquetas de la categoría "roles sociales"

\section{Conocimiento compartido}

\begin{tabular}{|c|l|}
\hline Categoria & \multicolumn{1}{|c|}{ Explicación } \\
\hline Correcto & $\begin{array}{l}\text { El/la hablante emite información nueva, no presupone información } \\
\text { personal que el interlocutor no conoce y no repite información que ya } \\
\text { ha mencionado. } \\
\text { Naci en Santander, en el 36. }\end{array}$ \\
\hline Sin referente & $\begin{array}{l}\text { Emite información desconocida para la entrevistadora como si ya } \\
\text { fuese conocida... } \\
\text { Yentonces le dije a Lolita: cpero qué dices? }\end{array}$ \\
\hline Repetición & $\begin{array}{l}\text { Introduce como nueva información que ya ha dado a la entrevistadora. } \\
\text { INF: Nací en Santander, en el 36. ENT:- ¿ Y qué te gustaba más de vivir } \\
\text { en Santander? INF: - ¿Santander? Yo nací alli, en el 36. }\end{array}$ \\
\hline Irrelevante & $\begin{array}{l}\text { Emite explicaciones o hace preguntas básicas sobre conocimiento del } \\
\text { mundo. } \\
\text { ¿Sabes lo que es el espacio? }\end{array}$ \\
\hline Incomprensible & $\begin{array}{l}\text { Emite enunciados incomprensibles para la entrevistadora. } \\
\text { Si... Yo, mi piso... Y estoy... Sí, sí... Algunas y... Otras pues no van o } \\
\text { vienen. } .\end{array}$ \\
\hline Contradicción & $\begin{array}{l}\text { Emite información contradictoria a la que ha transmitido previamente } \\
\text { al interlocutor. } \\
\text { ENT: Pero yo soy la mayor [...] Tengo [hermanos]: Rosa. Y que es la } \\
\text { mayor. Yo soy la-la segunda }\end{array}$ \\
\hline
\end{tabular}

Tabla 5. Etiquetas de la categoría "conocimiento compartido"

\subsection{TRATAMIENTO DE DATOS}

Por último, se procedió a analizar cuantitativamente los datos obtenidos tras el proceso de anotación para poder así cumplir el objetivo (iii) de este trabajo (comprobar si la capacidad de las personas con demencia para introducir la información contextual correctamente se reduce conforme avanza el deterioro cognitivo). Se realizaron los dos siguientes cálculos estadisticos mediante el software IBM SPSS Statistics 23:

- Coeficientes de correlación de Pearson $(r)$, a través del cual se determina la relación lineal entre dos variables (Murgiondo y Tejedo, 2005).

- Coeficiente de determinación $\left(r^{2}\right)$, que muestra el porcentaje de variabilidad que resulta al poner en relación dos variables (Pita Fernández y Pértega Diaz, 1997).

Las variables empleadas en estos cálculos fueron: por un lado, el porcentaje de usos anotados como correctos para cada una de las cuatro categorías de análisis (lugar, tiempo, roles sociales y conocimiento compartido) $\mathrm{y}$, por otro, el estadio de la enfermedad. 


\section{Resultados}

\subsection{LUGAR}

Los resultados de este apartado indican que existe una correlación negativa muy fuerte entre el estadio de la enfermedad (GDS-3 y GDS-4= leve, GDS-5= moderado; GDS-6 y GDS-7=severo) $(\mathrm{M}=1.70 ; \mathrm{SD}=.82)$ y el número de referencias de lugar correctas $(\mathrm{M}=.64 ; \mathrm{SD}=.37), r=-.909, p=000, \mathrm{n}=23$, con un coeficiente de determinación $R_{2}=.827$. En lo que respecta al porcentaje de referencias de lugar correctas, este descendió del 91\% en el estadio leve $(n=7)$, al $56,5 \%$ en el moderado $(n=6)$ y al $17,4 \%$ en el severo $(n=5)$. En los tres estadios la respuesta incorrecta más común fue la referencia de lugar sin referente $(3,6 \%$ de las respuestas en el leve, $17,6 \%$ en el moderado y $44,2 \%$ en el severo), seguido de la referencia incorrecta (3\%, 14,8\% y 32,6\% respectivamente). La Tabla 6 muestra el porcentaje de tipo de referencia de lugar según el GDS y los estadios de la enfermedad.

\begin{tabular}{cccccc}
\hline Estadio & Correcto & Incorrecto & Sin referente & No especifico & Contradictorio \\
\hline INICIAL (3-4) & $91 \%$ & $3 \%$ & $3,6 \%$ & $2,3 \%$ & $0 \%$ \\
\hline MODERADO (5) & $56,5 \%$ & $14,8 \%$ & $17,6 \%$ & $11,1 \%$ & $0 \%$ \\
\hline SEVERO (6-7) & $17,4 \%$ & $32,6 \%$ & $44,2 \%$ & $5,8 \%$ & $0 \%$ \\
\hline
\end{tabular}

Tabla 6: Porcentajes del análisis de lugar por estadio de la enfermedad

\subsection{TIEMPO}

Los resultados de este apartado indican que existe una correlación negativa muy fuerte entre el estadio de la enfermedad $(\mathrm{M}=1,70 ; \mathrm{SD}=0,82)$ y el número de referencias de tiempo correctas $(\mathrm{M}=.60 ; \mathrm{SD}=.38), r=-.857 p=000$, $\mathrm{n}=23$, con un coeficiente de determinación de $R^{2}=.735$. En lo que respecta al porcentaje de referencias correctas, este descendió del 88,7\% en el estadio leve $(n=7)$, al $68,7 \%$ en el moderado $(n=6)$ y al $25 \%$ en el severo $(n=5)$. En el estadio leve, la respuesta incorrecta más común fue la referencia de tiempo no específica $(8,1 \%)$. En el caso del estadio moderado fue la respuesta incorrecta $(14,9 \%)$, seguida de la no específica (9\%). En el estadio severo los errores más comunes fueron las referencias incorrectas $(37,5 \%)$, seguidas de las referencias sin referente $(25 \%)$. La Tabla 7 muestra el porcentaje de tipo de referencia de lugar según el GDS y los estadios de la enfermedad.

\begin{tabular}{cccccc} 
Estadio & Correcto & Incorrecto & Sin referente & No especifico & Contradictorio \\
\hline INICIAL (3-4) & $88,7 \%$ & $2 \%$ & $1 \%$ & $8,1 \%$ & $0,2 \%$ \\
\hline MODERADO(5) & $68,7 \%$ & $14,9 \%$ & $4,5 \%$ & $9 \%$ & $3,0 \%$ \\
\hline SEVERO (6-7) & $25 \%$ & $37,5 \%$ & $25 \%$ & $12,5 \%$ & $0 \%$ \\
\hline
\end{tabular}


Tabla 7: Porcentajes del análisis de tiempo por estadio de la enfermedad

\subsection{ROLES SOCIALES}

Los resultados indican que existe una correlación negativa fuerte entre el estadio de la enfermedad $(\mathrm{M}=1,70 ; \mathrm{SD}=0,82)$ y el porcentaje de referencias correctas a los roles sociales $(\mathrm{M}=.64 ; \mathrm{SD}=.45), r=-.733, p=000, \mathrm{n}=23$, con un coeficiente de determinación de $R^{2}=.538$. En lo que concierne al porcentaje de referencias correctas, este descendió del 91,6\% en el estadio leve ( $n=7)$, al $46,8 \%$ en el moderado $(n=6)$ y al $10 \%$ en el severo $(n=5)$. En los tres estadios la respuesta incorrecta más común fue el enunciado no relevante, pues supuso un 5,9\% de las respuestas en el leve, 14,3\% en el moderado. En el caso del estadio moderado fueron las respuestas incorrectas $(16,3 \%)$, seguida de la no específica (13,6\%). En el estadio severo los errores más comunes fueron tanto las referencias incorrectas $(5,7 \%)$ como las referencias sin referente $(5,7 \%)$. La Tabla 8 muestra el porcentaje de cada tipo de etiquetas de roles sociales según el GDS y los estadios de la enfermedad.

\begin{tabular}{cccc}
\hline Estadio & Correcto & Irrelevante & Incorrecto \\
\hline INICIAL (3-4) & $90,9 \%$ & $2,1 \%$ & $2,1 \%$ \\
\hline MODERADO(5) & $39 \%$ & $44,4 \%$ & $0 \%$ \\
\hline SEVERO (6-7) & $10 \%$ & $11,4 \%$ & $18,6 \%$ \\
\hline
\end{tabular}

Tabla 8: Porcentajes del análisis de roles sociales por estadio de la enfermedad

\subsection{CONOCIMIENTO COMPARTIDO}

Los resultados indican que existe una correlación negativa entre el estadio de la enfermedad $(\mathrm{M}=1,70 ; \mathrm{SD}=0,82)$ y el uso correcto de la información compartida. $(\mathrm{M}=.47 ; \mathrm{SD}=.38), r=.580, p \leq .004, \mathrm{n}=23$, con un coeficiente de determinación de $R^{2}=.337$. En lo que concierne al porcentaje de referencias correctas, este descendió del 82,8\% en el estadio leve (n=7), al 73,8\% en el moderado $(n=6)$ y al $32,7 \%$ en el severo $(n=5)$. En el estadio inicial el error más común fue emitir información ya conocida por la entrevistadora como si fuese nueva $(13,5 \%)$. Sin embargo, en los estadios moderado y severo fueron más habituales los enunciados en los que no se entendia qué información intentaba transmitir la persona entrevistada $(15,2 \%$ y $63,4 \%$ respectivamente). La Tabla 9 muestra el porcentaje medio de cada tipo de enunciado en conocimiento compartido por GDS y estadios de la enfermedad:

\begin{tabular}{ccccccc}
\hline Estadio & Correcto & Sin ref. & Repetición & Irrel. & Incompr. & Contradic. \\
\hline INICIAL (3-4) & $82,8 \%$ & $3,2 \%$ & $13,5 \%$ & $0,2 \%$ & $0,1 \%$ & $0,1 \%$ \\
\hline MODERADO (5) & $73,8 \%$ & $0,4 \%$ & $7,6 \%$ & $2,5 \%$ & $15,2 \%$ & $0,4 \%$ \\
\hline
\end{tabular}


SEVERO (6-7)

$32,7 \%$

$0,7 \%$

$2,6 \%$

$0 \%$

$63,4 \%$

$0,7 \%$

Tabla 9: Porcentajes del análisis de conocimiento compartido por estadio de la enfermedad

\subsection{ConteXto como conJunto}

En este apartado se muestran los resultados que tienen que ver con el análisis del contexto tomado en su conjunto, es decir integrando en un único análisis el escenario (lugar y tiempo) y los participantes (roles sociales y conocimiento compartido). En esta parte se han sumado los valores de la fracción de usos correctos en cada parte para crear una escala entre 0-4. Los datos resultantes indican que existe una correlación negativa fuerte entre el estadio de la enfermedad $(\mathrm{M}=1,70 ; \mathrm{SD}=0,82)$ y la capacidad para integrar la información contextual en los enunciados en las personas con demencia $(\mathrm{M}=2.3 ; \mathrm{SD}=1.4), r=.862, p=000, \mathrm{n}=23$, con un coeficiente de determinación de $R^{2}=.742$. Si atendemos a las diferencias entre estadios, observamos que la puntuación en los cuatro apartados (lugar, tiempo, roles sociales y conocimiento compartido) se reduce conforme aumenta el deterioro cognitivo. Esto se refleja en la puntuación total, de modo que del estadio leve ( $n=7$; 3,28 puntos) al moderado ( $\mathrm{n}=6 ; 2,25$ puntos) se produce un descenso del $26 \%$ y de este al severo ( $n=5 ; 0,23$ puntos) la caída es aún mayor, con una disminución del 51\%.

Esto nos indica que, en el caso de este corpus, el principal deterioro de las habilidades para integrar la información contextual para posibilitar la emisión de enunciados pragmáticamente coherentes acontece cuando las personas que padecen demencia se encuentran entre el GDS-4 y el GDS-6. La Tabla 10 muestra las puntuaciones medias de nuestros informantes en contexto según estadio y grado de GDS.

\begin{tabular}{cccccc}
\hline Estadio & Lugar & Tiempo & Roles & Con. Comp. & Total \\
\hline INICIAL (3-4) & 0,91 & 0,86 & 0,91 & 0,60 & 3,28 \\
\hline MODERADO (5) & 0,57 & 0,53 & 0,56 & 0,59 & 2,25 \\
\hline SEVERO (6-7) & 0,07 & 0,06 & 0,10 & 0 & 0,23 \\
\hline
\end{tabular}

Tabla 10: Medias de la anotación en cada apartado de "contexto" por estadio

\section{Discusión Y CONCLUSIONES}

La falta de consenso en torno a la definición de contexto en el campo de la pragmática clínica, ya resaltada por autores como Cummings (2012), ha hecho que la mayoria de las investigaciones que se han llevado a cabo sobre pragmática y demencia hayan partido de perspectivas teóricas diferentes. Con esto en mente, en esta investigación propusimos como primer objetivo "proponer un modelo de análisis de la integración de información contextual en el discurso". Para ello, tomamos como punto de partida el modelo de Van Dijk y lo simplificamos para poder aplicarlo al análisis del discurso de una 
persona con demencia. Esta adaptación nos resultó muy útil y cubrió de forma eficaz nuestras necesidades a la hora de realizar un análisis de la integración de la información contextual por parte de una persona con demencia.

Además, el segundo objetivo de este trabajo era "identificar cuáles son los patrones de error de las personas con demencia cuando integran la información contextual en su discurso". Para ello, propusimos una subcategorización de cada uno de los cuatro elementos que examinamos: tiempo, lugar, roles sociales y conocimiento compartido. Se trata de una propuesta que surgió de nuestras necesidades de anotación y que ha funcionado en el caso de nuestro corpus. Esperamos que esta propuesta sea contrastada, completada y mejorada en futuros trabajos que cuenten con un corpus con una casuística distinta.

Aparte de esto, los trabajos que conocemos hasta ahora sobre contexto y demencia, de los cuales hemos hablado detalladamente en $\S 2$, se habian centrado sobre todo en cómo la información contextual puede actuar como coadyuvante para continuar con las interacciones de forma exitosa. Sin embargo, esta investigación se ha propuesto como tercer objetivo "comprobar si la capacidad de las personas con demencia para introducir la información contextual correctamente se reduce conforme avanza el deterioro cognitivo" $y$, como consecuencia, ha presentado un enfoque nuevo en el que se ha analizado si se deteriora la capacidad de integrar los elementos contextuales adecuadamente en el discurso.

En el caso de la información que concierne a lugar, hemos detectado una correlación negativa muy fuerte $(r=-.909)$ entre el grado de deterioro cognitivo y el número de referencias correctas empleado en el discurso de las personas con demencia. Concretamente, el porcentaje de usos correctos descendió del $91 \%$ en el estadio leve ( $n=7$ ), al $56,5 \%$ en el moderado ( $n=6)$ y al $17,4 \%$ en el severo $(n=5)$. Este patrón de deterioro se asemeja al que propone la Global Deterioration Scale (GDS) con respecto a la orientación espacial. Según esta escala, en los estadios iniciales (hasta el grado 4) la orientación espacial permanece relativamente preservada, aunque las personas comienzan a presentar problemas para dirigirse a lugares desconocidos. En el grado 5, es decir, en el estadio moderado, la persona ya muestra déficits de desorientación espacial, y en el grado 6 esta desorientación ya es total. Esto nos permite afirmar que el deterioro en el uso de las referencias espaciales se produce paralelamente al de la orientación espacial. Asimismo, el deterioro de la memoria, tanto a corto como a largo plazo, es otro de los factores que incide en la corrección de las referencias espaciales.

En el caso de la información relacionada con el tiempo, existe una correlación negativa muy fuerte entre el estadio de la enfermedad y el uso correcto de las referencias temporales $(r=-.857)$. El porcentaje de usos correctos descendió del $88,7 \%$ en el estadio leve, al $68,7 \%$ en el moderado y al $25 \%$ en el severo. Tal y como sucede con las referencias espaciales y la orientación espacial, en el caso de las temporales también se detecta un paralelismo con 
el patrón que se muestra en la GDS en lo que concierne a la orientación temporal: se mantiene preservada en los grados iniciales (3 y 4), el deterioro comienza en el grado moderado (5) y a partir del grado 6 el deterioro es completo. Al igual que en el caso anterior, los problemas de memoria también dificultan la emisión de enunciados con referencias temporales correctas.

En lo que concierne a los roles sociales, se ha determinado una correlación negativa fuerte entre el estadio de la enfermedad y el porcentaje de referencias correctas $(r=-.733)$. Este porcentaje descendió del $68,1 \%$ en el estadio leve al $25 \%$ en el moderado y al $13 \%$ en el severo. Con respecto a los roles sociales, podemos relacionar el deterioro con la orientación personal, que de nuevo sigue el patrón determinado por la escala GDS, en la que se indica que esta se encuentra relativamente preservada hasta el estadio moderado (grado 5) y totalmente dañada a partir del grado 6. Igualmente relacionada está la memoria a corto plazo, pues a todas las personas informantes se les explicó antes de comenzar la grabación que iban a ser grabadas para un estudio de la universidad, pero varios de los errores en este apartado se debieron a que no recordaban cuál era el rol de la persona que realizaba la entrevista.

Con respecto al conocimiento compartido, cabe señalar que su análisis resultó más complejo que el de los otros elementos de análisis del contexto. Esto es debido a que, al tratarse de una entrevista, la persona que realizaba las preguntas proponía temas nuevos que favorecían que la persona entrevistada no repitiese información anterior, por lo que sería conveniente realizar esta misma prueba con otros tipos de narración para comprobar si los resultados son similares a los nuestros. En este caso, los datos indican que existe una correlación negativa moderada entre el estadio de la enfermedad y el uso correcto de la información compartida. $(r=-.580)$. Es conveniente destacar que el deterioro entre estadios fue menos progresivo que en los otros elementos de análisis del contexto, pues el uso correcto del conocimiento compartido apenas descendió del $82,8 \%$ en el estadio leve al $73,8 \%$ en el moderado. El descenso más pronunciado fue entre este último y el severo $(32,7 \%)$. En este caso, el deterioro lingüístico está relacionado también con los problemas de orientación personal y de memoria a corto y largo plazo.

Si observamos la situación desde una perspectiva de conjunto, los resultados obtenidos indican que existe una correlación negativa fuerte $(r=-.862)$ entre el grado del deterioro cognitivo y la capacidad de las personas que padecen demencia para integrar la información contextual en sus interacciones. Lógicamente, estos problemas causan un impacto reseñable en la coherencia pragmática del discurso y dificultan que, en numerosas ocasiones, los interlocutores de aquellas personas en estadio más avanzado puedan ubicar temporal, espacial y personalmente el contenido de los enunciados y, por lo tanto, tendrán más problemas para comprender el discurso. 
Teniendo todo esto en cuenta, en futuras investigaciones buscaremos plantear material para que las personas en los estadios iniciales de la demencia puedan trabajar estos aspectos en talleres de rehabilitación cognitiva y, por lo tanto, puedan intentar preservar la capacidad de integrar información contextual correctamente el máximo tiempo posible. Asimismo, sería muy enriquecedor que futuros trabajos aplicasen el modelo de análisis de contexto que hemos planteado en este artículo en otro tipo de corpus, bien sea con narraciones de otro tipo o con otras poblaciones que también presenten trastornos pragmáticos del lenguaje (por ejemplo, personas con esquizofrenia o autismo). Esto permitiría contrastar y mejorar el modelo que aquí se plantea.

\section{REFERENCIAS}

ASP, E. D. \& DE VILLIERS, J. (2010): When Language Breaks Down: Analysing Discourse in Clinical Contexts, Cambridge: Cambridge University Press.

CHENERY, H. J.; INGRAM, J. C. \& MURDOCH, B. E. (1990): “Automatic and volitional semantic processing in aphasia”, Brain and Language, 38 (2), pp. 215232.

CUMMINGS, L. (2012): "Theorising context", Finkbeiner, R.; Meibauer, J.; Shcumacher, P. B. (eds.): What is a Context?: Linguistics Approaches and Challenges, Amsterdam and Philadelphia: John Benjamins, pp. 55-80.

HAMILTON, H. E. (1994): Conversations with an Alzheimer's patient: An interactional sociolinguistic study, Cambridge: Cambridge University Press.

JOUBERT, S.; MAURIES, S.; BARBEAU, E.; CECCALDI, M. \& PONCET, M. (2004): "The role of context in remembering familar persons: insights from semantic dementia", Brain and cognition, 55 (2), pp. 254-261.

MÜLLER N. (ed.) (2006): Multilayered transcription, San Diego, CA: Plural Publishing.

MURGIONDO, J. E. y TEJEDOR, F. J. T. (2005): Análisis descriptivo de datos en educación, 2 Madrid: La Muralla.

NEBES, R. D. \& HALLIGAN, E. M. (1996): "Sentence context influences the interpretation of word meaning by Alzheimer patients", Brain and Language, 54 (2), pp. 233-245.

ORGANIZACIÓN MUNDIAL DE LA SALUD (OMS) Demencia. Disponible en: http:/ / www.who.int/mediacentre/factsheets/fs362/es/ (Fecha de consulta 03/09/ 2017).

PÉREZ MANTERO, J. L. (2017): Descripción y análisis pragmático del lenguaje en la demencia de tipo alzhéimer, València: Universitat de Valência.

PITA FERNÁNDEZ, S. y PÉRTEGA DÍAZ, S. (1997): "Relación entre variables cuantitativas", Cadernos de Atención Primaria, 4, pp. 141-144.

RAMANATHAN-ABBOTT, V. (1994): "Interactional differences in Alzheimer's discourse: An examination of $\mathrm{AD}$ speech across two audiences", Language in Society, 23 (1), pp. 31-58.

RAMANATHAN, V. (1995): "Interactional differences in Alzheimer discourse", Language in Society, 1, pp. 31-58. 
VARELA SUÁREZ, A. (2018): Lengua y demencia: Diseño y aplicación de un protocolo de coherencia discursiva (Tesis doctoral, Universidade de Vigo).

VAN DIJK, T. A. (2012): Discurso y contexto: un enfoque sociocognitivo, Barcelona: Gedisa.
VAN DIJK, T. A. \& KINTSCH, W. (1983): Strategies of Discourse Comprehension, New York: Academic Press. 